\title{
Clinical investigation: An endangered science
}

A decade ago, James B. Wyngaarden, a former director of the US National Institutes of Health (NIH), struck a resonant chord in the world of biomedical research when he wrote about the physician-scientist as an "endangered species". Throughout the history of medicine, physicians who applied their intellectual talents to the observation of patients were the ones who made the most important discoveries about health and disease. Aristotle, who studied both animals and humans, identified and named the aorta, becoming the father of anatomy and paving the way for the later work of Andreas Vesalius whose 16th century drawings of the human body remain among the most important contributions to medicine.

In our own time, the majority of advances in understanding human physiology came from research on patients, and particularly in the United States, the physician-scientist became the paradigm. The opening of the Clinical Center at NIH in 1952 is still considered a milestone in medicine. The Clinical Center, with patients' rooms on one side of the corridor and research laboratories on the other, represented the epitome of patientbased research; physicians came from all over the world to work there.

But time, inevitably, has brought change. The molecular revolution, ushered in with the discovery of recombinant DNA techniques in the early 1970s, has enabled scientists to make astonishing discoveries about the functions of genes and molecules without examination. The researcher with a $\mathrm{PhD}$ degree and substantial training in molecular biology became a major player in biomedical science, sometimes overshadowing the physician-scientist. But in the final analysis, the fruits of molecular research must still be tested on living patients and for this, the physician-scientist remains essential. That is why Wyngaarden and other biomedical leaders worried about their declining ranks.

Now, another force threatens the physician-scientist. Health-care reform, with its emphasis on money, is anathema to the support of clinical research which requires not only a specially trained cadre of physicians but also a pool of patients whose experimental care is bound to be expensive. It is difficult to imagine who will pay for future advances in medicine, given that the sole objective of health-care reform is to get patients in and out of the hospital as fast as possible.

Could Christian Barnard transplant a human heart and expect anyone to pay his patients' costs? If bone marrow transplantation were new, would the insurance companies of the 1990s be happy to foot the bill, knowing that much of the cost supported research? And what about chemotherapy, the development of which depended so significantly on NIH experimental protocols, paid for by federal research dollars.

Gene therapy is perhaps the most recent example of expensive clinical research that is now under economic threat because initial clinical trials, inevitably, are expensive. But with luck, that will not last.

Throughout the developed world, medicine is undergoing an economically driven cultural revolution in the name of health-care reform that is unprecedented. Last year in the United States, President Bill Clinton put health-care reform at the top of his policy agenda, only to see his monstrous legislative plan collapse under its own weight and complexity. Whether Clinton will produce a simpler agenda remains to be seen, but it is clear that the United States is not alone in its preoccupation with health-care reform. Canada, Britain, France, Germany, Sweden, The Netherlands - name almost any developed country in North America or Europe and you will find a nation beset with concern about reforming its medical system.

It is important to understand that health-care reform is not about health. It is about money. Driven by a compelling desire to cut costs, the marketplace (public or private) is subjecting medicine to change. The repercussions of this are quite predictable. The once sacred doctor-patient relationship is, perhaps, the most obvious. Until very recently, no one denied that a physician's primary moral and fiduciary responsibility was to the patient. But now, as more and more physicians find themselves working in managed care networks whose objective is to reduce costs, the emphasis has shifted towards saving money (often by eliminating services) and is in direct conflict with the notion that the good of the patient comes ahead of the networks' bottom line.

Health-care reform presents an equally serious threat to clinical research and its practitioners. Managed care companies do not want to pay for anything they don't have to, and government funding (with the exception of large clinical trials) is shifting to molecular research. It is important that the will and the means be found to preserve clinical research in a cost-conscious environment, especially one in which it is easy to be lulled into thinking that basic laboratory science has supplanted the need to study patients. One need look no further than the recent, astonishing discovery of a connective bridge of tissue between the spinal dura and rectus capitis posterior minor muscle at the base of the skull (pages 297-298) to see that research on real patients, whether living or recently deceased, is vital to human medicine.

Barbara J. Culliton 